Canadian Oncology

Nursing Journal

Revue canadienne

de soins infirmiers

en oncologie

Volume 31, Issue 3 • Summer 2021

elSSN: 2368-8076 


\title{
Factors influencing breast cancer screening practices among women of reproductive age in South Kayonza District, Rwanda
}

\author{
by Pierre Céléstin Igiraneza, Lilian A. Omondi, Bellancille Nikuze, Marie Goretti Uwayezu, Margaret Fitch, Gaudence Niyonsenga
}

\begin{abstract}
Introduction: Breast cancer in Rwanda is a major concern for women's health, as most breast cancer diagnoses are made at an advanced stage. Yet, diagnosis can be done early with breast cancer screening practices.
\end{abstract}

Aim: To assess factors influencing breast cancer screening practices among women of reproductive age in South Kayonza District of Rwanda.

Methodology: A quantitative survey was conducted with 246 women between 16 and 49 years of age from four health centres in South Kayonza District. A structured, pretested, and self-administered questionnaire was used to assess the influence of knowledge, health facilities, and socioeconomic and cultural factors on breast screening practices among women in South Kayonza.

\section{AUTHOR NOTES}
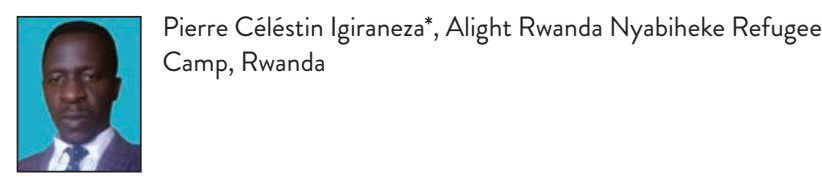

Pierre Céléstin Igiraneza*, Alight Rwanda Nyabiheke Refugee Camp, Rwanda

Lilian A. Omondi, School of Nursing and Midwifery, University of Rwanda, College of Medicine and Health Sciences, Kigali, Rwanda, New York University, Rory Meyers College of Nursing, and School of Nursing Sciences, University of Nairobi, Kenya

Bellancille Nikuze, School of Nursing and Midwifery, University of Rwanda, College of Medicine and Health Sciences, Kigali, Rwanda, New York University, Rory Meyers College of Nursing, and School of Nursing Sciences, University of Nairobi, Kenya

Marie Goretti Uwayezu, School of Nursing and Midwifery, University of Rwanda, College of Medicine and Health Sciences, Kigali, Rwanda, New York University, Rory Meyers College of Nursing, and School of Nursing Sciences, University of Nairobi, Kenya

Margaret Fitch, Rory Meyer's College of Nursing, New York University, New York, USA, and the Bloomberg Faculty of Nursing, University of Toronto, Canada

Gaudence Niyonsenga, King Faisal Hospital, Kigali. Rwanda

${ }^{*}$ Corresponding author: Pierre Céléstin Igiraneza, Alight Rwanda Nyabiheke Refugee Camp

Email: igiraneza7@gmail.com. Phone: +250788772820

DOI:10.5737/23688076313251257
Result: Among all respondents, 55.5\% had moderate knowledge about breast cancer risk factors and $60.2 \%$ also had moderate knowledge on the influence of breast cancer screening. While $28 \%$ were knowledgeable about Breast Self-Examination (BSE), 16.4\% were knowledgeable about Clinical Breast Examination (CBE) and breast ultrasound. Responses about the practice of breast screening in the last two years showed $72 \%$ of respondents had never practiced BSE and $81.3 \%$ had not used CBE or breast ultrasound scan. The main source of information about breast cancer screening was the radio, used by $30.4 \%$ of respondents. Significant relationships were found between age and marital status with breast cancer screening practices.

Conclusion: This study revealed low levels of knowledge about breast cancer, breast cancer screening, and practice regarding breast cancer screening. There is a need to promote awareness of breast cancer and create a program, which can influence breast cancer screening knowledge and practice to ultimately improve health among women in south Kayonza.

Key words: breast cancer screening, influence, practice.

\section{BACKGROUND}

lobally, in 2018, it was estimated that 627,000 women died $\checkmark$ from breast cancer accounting for approximately 15\% of all cancer deaths among women (Bray et al., 2018) . Breast cancer was considered for many years as primarily a disease of developed countries, whereas now higher mortality is being reported in developing countries. The five-year survival rate in females with newly diagnosed breast cancer in the United States has extended to $89 \%$, but it is estimated that the rate is $40 \%$ in developing countries (Morse et al., 2014). Annually, worldwide approximations of new cases of breast cancer being diagnosed is 1.7 million. It is estimated that $60 \%$ of mortality rates of women with cancer are caused by breast cancer with most of them occurring in developing countries; every year around 249,260 new cases of breast cancer are confirmed in developing countries. The cases in developing countries represents one-half of all breast cancer and $62 \%$ of breast cancer mortality rates (Tfayli et al., 2010).

Breast cancer remains the leading cause of death among women in Africa. Overall, an estimated 882,900 women in developing countries were diagnosed, of which 324,300 died, with the higher prevalence rates noted in East, North, and West Africa (Donkor et al., 2015). Approximations of standardized age prevalence rates per 100,000 women were 30.4 in Eastern Africa, 26 in Central Africa, 38.6 in Western Africa, and 38.9 in Southern Africa (Donkor et al., 2015). In sub-Saharan Africa, it is estimated that about 94,000 women are diagnosed with 
breast cancer and 48,000 of them die due to the disease each year. In East Africa, GLOBOCAN estimated that new breast cancer cases were $19.9 \%$ in females of all ages; incidence rates per 100,000 in Kenya and Uganda were 52\% and 34\% respectively (Cumber et al., 2017). As a country-specific example, the Globocan 2020 data show that, in Kenya, breast incidence rate is estimated at 40.3 per 100,000 women with a mortality rate of 17.8 per 100,000 . Annually, breast cancer incidence in Kenya is about $12 \%$ of all new cancer cases and mortality rate is about 7.7\% of all cancer deaths (Word Health Organization, 2020).

The Globocan 2020 data show that, in Rwanda, breast incidence rate is estimated at 41.0 per 100,000 women with a mortality rate of 19.4 per 100,000 . Annually, breast cancer incidence in Rwanda is $16.1 \%$ of all new cancer cases (World Health Organization, 2020). A study completed in Butaro Cancer Center of Excellence in Rwanda revealed that, of the $82 \%$ of women diagnosed with breast tumours, $55 \%$ of those were diagnosed with breast cancer and 36\% were diagnosed with benign tumours. For the women diagnosed with breast cancer, approximately $20 \%$ were stage one or two at diagnosis, $46 \%$ had locally progressive disease, and $31 \%$ had metastatic breast cancer (Pace et al., 2016).

Early detection of changes in the breast and prompt intervention results in better prognosis, decreased morbidity, and reduced mortality related to breast cancer. The American Cancer Society provided strategies for primary detection of breast cancer through screening practices according to female ages (Majidi et al., 2017). Understanding of the factors influencing women in Rwanda about breast screening is limited. Therefore, the purpose of this study was to assess factors influencing breast cancer screening practices among women of reproductive age in South Kayonza District.

\section{MATERIAL AND METHODS Design}

This was a descriptive cross-sectional study of women aged 16-49 years in four selected Health Centers of Rwinkwavu catchment area, South Kayonza District, Rwanda. The catchment area of Rwinkwavu District Hospital is in the southern part of Kayonza District, located in the eastern province of Rwanda and is populated by 211,251 people. The economy of Kayonza District is mostly grounded on agriculture. The local growing is dominated by banana $(29 \%)$, maize $(16 \%)$, beans $(24 \%)$, cassava $(12 \%)$, and vegetables $(4 \%)$, while the main livestock is cattle. However, in addition to locally produced food, the population have geographical access to food brought from other parts of Rwanda through road transport where they are sold in different markets and trade stores (National Institute statistic of Rwanda, 2012).

\section{Sample size and sampling}

The sample size was calculated by using Fisher et al. (1998) formula where: $\mathrm{Z}=$ the standard normal deviate 1.96 at $95 \%$ confidence interval; $\mathrm{P}=$ estimated prevalence of mothers in the Rwinkwavu catchment area at $20 \%$; and $\mathrm{d}=$ precision of errors at $5 \%$. The calculated sample size required was 246 women.

\section{Data collection procedure}

Data collection occurred over two months. The principal researcher chose one assistant registered nurse in each health facility and provided training on how the research instrument was to be administered. The researcher and research assistants approached women at four selected health centres to participate in the study. The women attending the health centres for consultation as outpatients were requested to participate. After disclosure about the study, women who consented to participate and signed the consent form were interviewed by the assistant who administered the structured questionnaire. The assistant asked the questions and then recorded the women's answers. It took approximately 10 minutes to complete each questionnaire. The assistant submitted the completed questionnaires and signed informed consent forms to the researcher.

\section{Study instrument}

The data collection instrument was adapted for this study by the researcher after being reviewed with a team of Rwandan experts based at the University of Rwanda in School of Nursing and Midwifery. It was used previously by researchers in Nigeria and Kenya (Paul, 2012) and adapted for the Rwandan context. The detailed questionnaire used in this study consisted of five sections: section A: socioeconomic variables; section B: knowledge on breast risk factors, what factors influence breast screening practices, and breast cancer screening methods; section C: source of information on breast cancer; section D: health facilities influence on breast screening practices; and section E: socioeconomic and culture factors that prevent women from performing breast screening.

The assessment of the instrument face and content validity was done by using a pilot test involving 25 respondents from four health centres and consulting experts in the field of oncology nursing. This was done to make sure the research instrument measured what it was expected to measure by reviewing each question of the questionnaire and observing whether the whole data instrument was appropriate or not appropriate. The pilot questionnaires were distributed, and participants were invited to give their views about the guidance and precision of the questions. These views were incorporated to further refine the questionnaire. The pilot test data obtained were analyzed using Statistical package for social sciences (SPSS) version 21.0 and the reliability coefficient was 0.8 .

\section{Data analysis}

The data from the study sample were transformed into meaningful information for easy interpretation and understanding. The data were grouped, organized, ordered, and recorded into a cording sheet. Analysis using descriptive and inferential statistics was done using the computer program SPSS version 21.0 and presented in the form of charts and tables. Frequencies were calculated for all question responses and knowledge scores calculated by adding relevant item scores. A knowledge mean score above $80 \%$ was regarded as having 'good' knowledge, a knowledge score between $50 \%$ to $80 \%$ as 'moderate' knowledge, and scores less than $50 \%$ as 'poor' knowledge. 


\section{Ethical considerations}

Authorization to conduct research was obtained from the Institution Review Board (IRB) of the University of Rwanda, College of Medicine and Health Sciences. Authorization to collect data was obtained from the Director General of Rwinkwavu District Hospital. The purposes and objectives of the research were explained to each woman, as well as their rights to withdraw from the study, privacy, and anonymity. An informed consent form was signed prior to data collection to show women's agreement to participate in the study.

\section{RESULTS}

\section{Demographic characteristics}

The respondents were divided among age groups between 16 and 49 years (Table 1). About a third of respondents (32.9\%) were in the age group 30-39 years. The least percentage, 7.7\%, was in the $40-49$ years group. Whereas $61.8 \%$ of the women surveyed attended only primary education, $14.2 \%$ did not have basic education. A total of $24 \%$ attended secondary or university education. About half (56.1\%) of the women surveyed were in category three of Ubudehe (Population categorization on practice and culture of collective action and mutual support to solve problems within the community). Those categorized in category 2 and 1 were $33.3 \%$ and $10 \%$ respectively. In addition, $77.6 \%$ of women used community health-based insurance.

The study revealed that $34.1 \%$ of respondents surveyed were not confident in breast screening. While $32.6 \%$ of respondents had had clinical breast examinations (CBE) and ultrasound (US), $81.3 \%$ of respondents were not confident about CBE and ultrasound scan. Within the last two years, 200 respondents had never practised breast self-examination (BSE), representing $81.3 \%$ of the total respondents; $14.6 \%$ of the total number of respondents had BSE once a year (Table 1).

\section{Knowledge regarding breast cancer risk}

About half (56.1\%) of the respondents surveyed were knowledgeable about breast cancer risk factors $(\mathrm{N}=246)$ (see Table 2). Those who were not knowledgeable about breast cancer risk factors were $43.9 \%$ of respondents. No statistically significant associations were found with knowledge and demographic variables.

Table 3 shows influences on women's breast cancer screening practices. Almost three-quarters (74.4\%) of the respondents surveyed agreed that abnormal discharge should influence them to undertake CBE or ultrasound scan and $70.7 \%$ of respondents agreed that a breast mass and pain should influence them. Only $39.9 \%$ of respondents were influenced by the failing of traditional healing applications on breast tumours and the applications not having the desired effect.

Most of the respondents $(60.2 \%)$ had moderate knowledge about influences of breast cancer screening, while $55.5 \%$ had moderate knowledge about breast cancer risk factors. There was poor knowledge about BSE (20.8\%) and ultrasound scans for $16.5 \%$, (Figure 4.1 ).

\section{Breast screening practices among respondents}

Approximately one-third of the women in each age group between 16 and 39 years engaged in breast screening (Table 1). Only $7.7 \%$ of those in the $40-49$-year age group were screened.

\begin{tabular}{|c|c|c|}
\hline Demographic categories & Frequency & Percent (\%) \\
\hline \multicolumn{3}{|l|}{ Age } \\
\hline $16-19$ & 70 & 28.5 \\
\hline $20-29$ & 76 & 30.9 \\
\hline $30-39$ & 81 & 32.9 \\
\hline $40-49$ & 19 & 7.7 \\
\hline \multicolumn{3}{|l|}{ Education level } \\
\hline No basic education & 35 & 14.2 \\
\hline Primary & 152 & 61.8 \\
\hline Secondary & 50 & 20.3 \\
\hline University level & 9 & 3.7 \\
\hline \multicolumn{3}{|l|}{ Marital status } \\
\hline Single & 58 & 23.6 \\
\hline Married & 153 & 62.2 \\
\hline Separated & 18 & 7.3 \\
\hline Widowed & 17 & 6.9 \\
\hline \multicolumn{3}{|c|}{ Ubudehe (social categories) } \\
\hline Category 1 & 26 & 10.6 \\
\hline Category 2 & 82 & 33.3 \\
\hline Category 3 & 138 & 56.1 \\
\hline Category 4 & 0 & 0 \\
\hline \multicolumn{3}{|l|}{ Health insurance } \\
\hline Others & 7 & 2.8 \\
\hline $\mathrm{CHBI}$ & 191 & 77.6 \\
\hline RAMA & 28 & 11.4 \\
\hline MMI & 8 & 3.3 \\
\hline UAP & 3 & 1.2 \\
\hline NONE & 9 & 3.7 \\
\hline \multicolumn{3}{|c|}{$\begin{array}{l}\text { Engage in breast screening (by age } \\
\text { group) }\end{array}$} \\
\hline $16-19$ & 20 & 28 \\
\hline $20-29$ & 76 & 30.9 \\
\hline $30-39$ & 81 & 32.6 \\
\hline $40-49$ & 19 & 7.7 \\
\hline \multicolumn{3}{|l|}{ Confidence performing } \\
\hline Confident & 43 & 17.5 \\
\hline Not confident & 84 & 34.1 \\
\hline \multicolumn{3}{|l|}{ Had CBE/US } \\
\hline At least once & 36 & 14.6 \\
\hline Never & 200 & 81.3 \\
\hline Unsure & 10 & 4.1 \\
\hline
\end{tabular}




\begin{tabular}{|l|c|c|c|c|c|}
\hline \multicolumn{2}{|l|}{ Table 2. Knowledge on breast cancer risk factors } \\
\hline $\begin{array}{l}\text { Knowledge about breast } \\
\text { cancer risk factors }\end{array}$ & Strongly disagree N (\%) & Disagree N (\%) & Neutral N (\%) & Agree N (\%) & Strongly agree N (\%) \\
\hline 1. Physical inactivity & $30(12.2)$ & $46(18.7)$ & $52(21.1)$ & $106(43.1)$ & $12(4.9)$ \\
\hline 2. Use of alcohol & $8(3.3)$ & $34(13.8)$ & $35(14.2)$ & $133(54.1)$ & $36(14.6)$ \\
\hline 3. Breast feeding & $9(3.7)$ & $57(23.2)$ & $31(12.6)$ & $112(45.5)$ & $37(15.0)$ \\
\hline 4. Witchcraft & $8.1(20)$ & $66(26.8)$ & $45(18.3)$ & $85(34.6)$ & $30(12.2)$ \\
\hline 5. Smoking & $13(5.3)$ & $46(18.7)$ & $21(8.5)$ & $119(48.4)$ & $47(19.1)$ \\
\hline 6. Breast cancer is inherited & $23(9.3)$ & $57(23.2)$ & $38(15.4)$ & $98(39.8)$ & $30(12.2)$ \\
\hline 7. Injury of breast & $17(6.9)$ & $50(20.3)$ & $30(12.2)$ & $109(44.3)$ & $40(16.3)$ \\
\hline $\begin{array}{l}\text { 8. Early onset of } \\
\text { menstruation }\end{array}$ & $30(12.2)$ & $77(31.3)$ & $29(11.8)$ & $77(31.3)$ & $33(13.4)$ \\
\hline Total & 7.6 & 22.0 & 14.3 & 42.6 & 13.5 \\
\hline
\end{tabular}

Table 3. Respondents' knowledge on influences of breast cancer screening practices

\begin{tabular}{|l|c|c|c|c|c|}
\hline VARIABLES & & & \\
\hline $\begin{array}{l}\text { Knowledge on influences of } \\
\text { breast cancer screening }\end{array}$ & Strongly disagree $n$ (\%) & Disagree $n$ (\%) & Neutral $n$ (\%) & Agree $n$ (\%) & Strongly agree $n$ (\%) \\
\hline 1. Having breast pain & $20(8.1)$ & $43(17.5)$ & $9(3.7)$ & $136(55.3)$ & $38(15.4)$ \\
\hline 2. Family history & $39(15.9$ & $74(30.1)$ & $14(5.7)$ & $84(34.1)$ & $35(14.2)$ \\
\hline 3. Breast mass & $20(8.1)$ & $45(18.3)$ & $7(2.8)$ & $130(52.8)$ & $44(17.9)$ \\
\hline 4. Abnormal discharges & $19(7.7)$ & $35(14.2)$ & $9(3.7)$ & $136(55.3)$ & $47(19.1)$ \\
\hline 5. Importance and value & $24(9.8)$ & $62(25.2)$ & $14(5.7)$ & $96(39.0)$ & $50(20.3)$ \\
\hline 6. Failure of herbal medicines & $30(12.2)$ & $103(41.9)$ & $15(6.1)$ & $73(29.7)$ & $25(10.2)$ \\
\hline Total & 10.3 & 24.5 & 4.6 & 44.4 & 16.2 \\
\hline
\end{tabular}

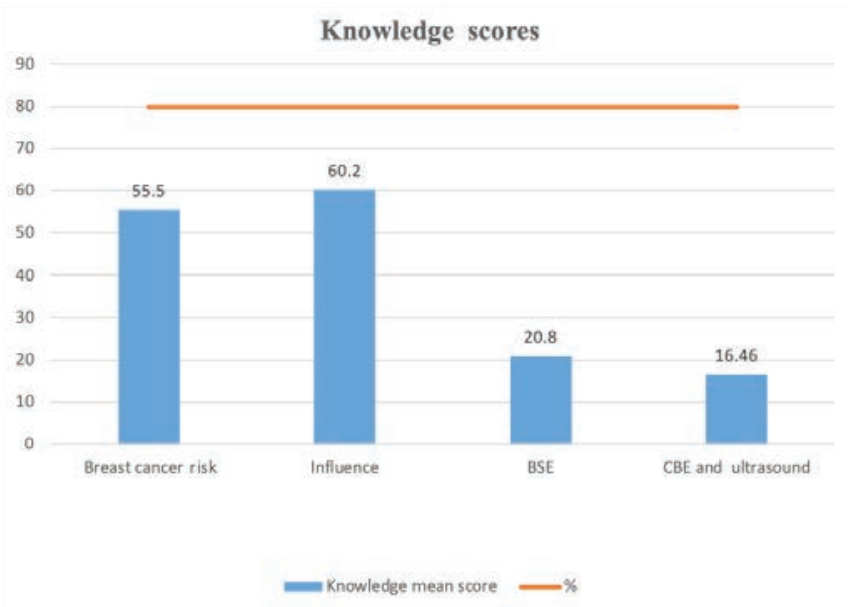

Figure 4.1. Knowledge scores

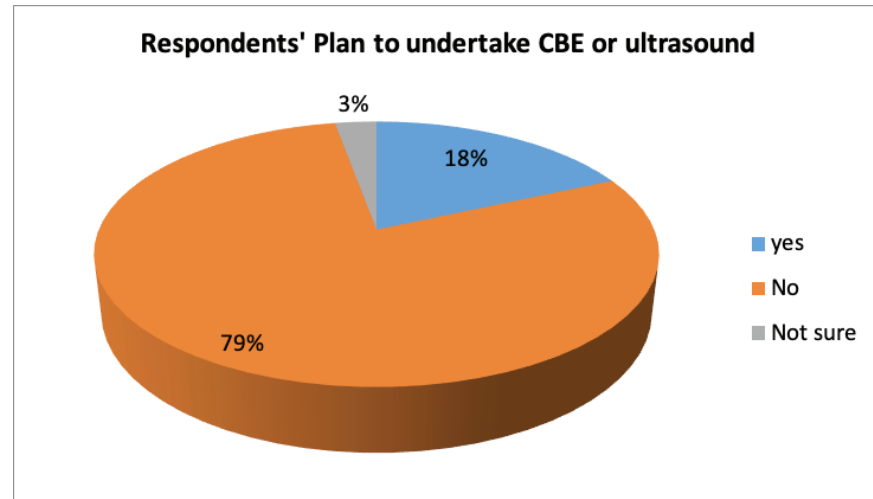

Figure 4.2. Plan to undertake clinical breast cancer examination and breast ultrasound scan 


\begin{tabular}{|c|c|c|c|}
\hline \multirow[t]{2}{*}{ VARIABLE } & \multicolumn{3}{|c|}{ BREAST SELF-EXAMINATION } \\
\hline & $\begin{array}{l}N(\%) \\
\text { df }\end{array}$ & Total (\%) & $p$ value \\
\hline \multicolumn{4}{|l|}{ Breast Health } \\
\hline \multicolumn{4}{|l|}{$\begin{array}{l}\text { Education at NCDs } \\
\text { clinic or maternity }\end{array}$} \\
\hline Provided & $\begin{array}{c}92(37.4 \%) \\
34.748^{a}\end{array}$ & $\begin{array}{c}246(100 \%) \\
12\end{array}$ & 0.001 \\
\hline Not provided & $154(62.6 \%)$ & & \\
\hline \multicolumn{4}{|l|}{$\begin{array}{l}\text { Provision of visual } \\
\text { aids at NCDs clinic or } \\
\text { maternity }\end{array}$} \\
\hline Provided & $\begin{array}{l}90(36.6) \\
45.597^{a}\end{array}$ & $\begin{array}{c}246(100 \%) \\
12\end{array}$ & 0.000 \\
\hline Not provided & $156(63.4 \%)$ & & \\
\hline \multicolumn{4}{|l|}{$\begin{array}{l}\text { Doctor and nurses' } \\
\text { advice about breast } \\
\text { screening }\end{array}$} \\
\hline Provided & $\begin{array}{c}48(19.5 \%) \\
6.521^{\mathrm{a}}\end{array}$ & $\begin{array}{c}246(100 \%) \\
12\end{array}$ & 0.008 \\
\hline Not provided & $198(80.5 \%)$ & & \\
\hline
\end{tabular}

A quarter $(25.6 \%)$ of the respondents were confident about doing BSE and $14.6 \%$ had ever had CBE or US of the breast. The majority of respondents (79\%) had no plan to undertake CBE or breast ultrasound scan while $8 \%$ did plan to undertake clinical breast examination. Only $3 \%$ of the respondents were not sure about undertaking CBE or breast ultrasound scan. See Figure 4.2.

\section{Influence of education at health facilities on breast cancer screening}

Table 4 shows that respondents who had breast health education at non-communicable diseases or maternity clinics were more likely to engage in breast cancer screening practices and this has a statistically significant association with breast screening practices $(n=34.748 ; p=0.001)$. Two-thirds $(62.6 \%)$ of the women who did not perform BSE had received breast health education at the health facilities. About $63.4 \%$ of the women who had not carried out BSE had never seen visual aids on breast cancer screening $=45.597, p=0.000$ ). Receiving advice on breast cancer screening has a statistically significant association with the practices of breast self-examinations ( $=45.597, p=0.008$ ). Eighty percent of respondents had not received advice about breast self-examination.

Seventy-two percent of the respondents agreed that the major barrier to breast cancer screening was lack of breast clinics (Table 5). Lack of knowledge about breast screening was also seen as a barrier for $49.2 \%$ of respondents and $48.8 \%$ agreed that long distance to health facilities was a barrier. There was no statistical significance between socioeconomic status and breast screening method.

Among the respondents who received information on breast health and breast cancer screening, 30.4\% of respondents obtained information on breast cancer and screening through the radio. In total, $35.4 \%$ of the respondents had no source of information on breast cancer and screening. Other sources of information were television (15\%), newspaper $(11.8 \%)$ friends $(0.8 \%)$, and hospitals $(1.2 \%)$.

\begin{tabular}{|l|c|c|c|c|c|}
\hline \multicolumn{2}{|l|}{ Table 5. Percentage and frequency of socio-economic and culture factors which are barriers to breast cancer screening practices } \\
\hline VARIABLES & Disagree strongly N (\%) & Disagree N (\%) & Neutral N (\%) & Agree N (\%) & Agree strongly N (\%) \\
\hline Culture practice & $58(23.6)$ & $133(54.1)$ & $26(10.6)$ & $28(11.4)$ & $1(0.4)$ \\
\hline Religious practice & $56(22.8)$ & $125(50.8)$ & $21(8.5)$ & $48(15.4)$ & $6(2.4)$ \\
\hline Social Stigma & $39(22.8)$ & $96(39)$ & $25(10.2)$ & $78(31.7)$ & $8(3.3)$ \\
\hline Lack of knowledge & $16(6.5)$ & $96(39.0)$ & $13(5.3)$ & $105(42.7)$ & $16(6.5)$ \\
\hline Long distance & $15(6.1)$ & $93(37.0)$ & $20(8.1)$ & $93(37.8)$ & $27(11.0)$ \\
\hline Lack of breast cancer clinic & $11(4.5)$ & $42(17.1)$ & $16(6.5)$ & $138(56.1)$ & $39(15.9)$ \\
\hline Cost of screening & $33(13.4)$ & $120(48.8)$ & $44(17.9)$ & $37(15.0)$ & $12(4.9)$ \\
\hline Fear of findings & $25(10.2)$ & $81(32.9)$ & $28(11.4)$ & $95(38.6)$ & $17(6.9)$ \\
\hline Ultrasound is painful & $44(17.9)$ & $120(48.8)$ & $37(15.0)$ & $34(13.8)$ & $11(4.5)$ \\
\hline Long waiting at health facilities & 25(10.2) & $78(31.7)$ & $17(6.9)$ & $97(39.4)$ & $29(11.8)$ \\
\hline
\end{tabular}




\section{DISCUSSION}

The results show that sociodemographic factors including social categories (Ubudehe), health insurance, education level, age, and marital status were significantly linked to breast selfbreast examination among women. The relationship between women's age categories and breast self- examination was statistically significant. The respondents within the age group 20-29 years were found to have the highest percent who engaged in breast self-examination practices, while women in the age category 40-49 years had the lowest percent. Being married had a positive significant link with practices of clinical breast examination and ultrasound breast scan. Breast screening was found to be more frequent in married women than single women, perhaps due to the improved familiarity about their accountability in being healthy enough and living longer to support her kids and entire family.

The study revealed that $55.5 \%$ of respondents have moderate knowledge scores on risk factors of breast cancer. The findings had same similarities with a Nigerian study which revealed that $84 \%$ women had poor knowledge, only $23 \%$ had fair knowledge, and $0.7 \%$ had good knowledge on breast cancer screening practices (Kohler et al., 2017a)many countries lack early detection services. In Malawi, women are frequently diagnosed with large tumors after long symptomatic periods. Little is known about local cancer knowledge. Methods We administered a cross-sectional survey with a discrete choice experiment to a random sample in urban and rural areas of Lilongwe district. Bivariable and multivariable analyses determined factors associated with knowledge. Preference utilities for early detection interventions were estimated using a hierarchical Bayesian model in Sawtooth software. Results Of 213 women recruited, fewer than half were aware of breast cancer. In multivariable analysis, electricity at home and knowing someone with cancer increased the odds of awareness. Women were more knowledgeable about symptoms than treatment or risk factors; more than $60 \%$ erroneously believed local misconceptions. Seventeen percent were aware of breast self-examination, and $20 \%$ were aware of clinical breast examination (CBE. This small difference might be related to sample characteristics in the two studies and different awareness of breast health.

Among all respondents, 68.7\% agreed that alcohol was a major risk factor followed by smoking 67.5\%. This study is inconsistent with an Ethiopian study regarding knowledge of respondents about risk factors: $71.3 \%$ of Ethiopian respondents agreed smoking was the most common risk factor and $44.2 \%$ of respondents agreed that alcohol consumption was a main risk factor (Tabrizi, 2015). This could be due to differences between sociodemographic and study areas.

Among respondents who had been taught breast self-examination, $81 \%$ of women surveyed confirmed they have never thought about breast self-examination. This study found that there was a significant relationship between education level and being taught about breast self-examination. Seventy-two percent of respondents had never practiced breast self-examination $=22.457, p=0.000$ ), whereas $17.5 \%$ practiced BSE once a year, $6.1 \%$ had carried out the examination once a month, and 4.1\% performed BSE once a week. More than three-quarters
(84.3\%) were not confident in BSE practices. The finding was higher than the findings in the study from the University of Buea where only $9.0 \%$ knew how to perform BSE, and only $3 \%$ had performed BSE regularly(Angahar, 2017). This difference could be due to differences in breast cancer awareness between the two study populations.

In our study, a majority of respondents $(81.3 \%)$ were not using clinical breast examination or breast ultrasound scan. Additionally, $79 \%$ had no plan to start CBE or obtain breast ultrasound scans in the next two years. This finding is consistent with a study conducted in Kenya in which $72 \%$ of women reported that they had not had clinical breast examination in the clinic (Tabrizi, 2015). This may be attributed to similar characteristics of the samples such as low levels of education, poverty, and deficiency in diagnostic test facilities. The two studies were done in rural areas and awareness of breast cancer screening was low compared to their counterparts in urban areas.

Among all respondents, $74.4 \%$ agreed that abnormal discharge can influence them to undertake CBE or breast ultrasound scan, followed by $70.7 \%$ of respondent who were influenced by breast mass and pain. Our findings are consistent with a study done by Ibrahim et al. (2013) who argues that women are influenced by having a lump in the breast, and a Nigerian study showing that respondents identified influences as bloody nipple discharge (21.1\%), breast lump symptoms (40.1\%), and breast pain (57\%) (Kohler et al., 2017b).

The study findings revealed that the respondents who received information on breast health, breast cancer, and screening used a variety of sources, but the primary one was the radio. These findings are similar to a study from Ghana where $39.8 \%$ of respondents agreed that radio was the major source of breast cancer information and other sources were television (17.5\%), family \& friends $(16.7 \%)$, newspapers and magazines (14.2\%). Only 5\% obtained their knowledge from doctors and nurses (Bray et al., 2018). The reasons for the low results could be due to the unavailability and inaccessibility of advertisements on breast cancer screening through radio, television, and newspaper. As well, the healthcare providers may not be providing effective health education on breast cancer screening modalities.

A study done in Egypt revealed that females with limited health literacy and lack of basic health information on breast cancer screening may have difficulties accessing facilities and understanding breast cancer-related skills (Bray et al., 2018). A study done in sub-Saharan Africa revealed that factors associated with lack of knowledge and insufficient health facilities are influencing the management of breast cancer (Angahar, 2017). Our results are in line with these findings as breast health education at NCDs or maternity clinics has a significant positive correlation with practicing BSE $\left(\chi^{2}=34.748, p=\right.$ $0.001)$. Additionally, $62.6 \%$ of the women who had not practiced breast-self-examination had not ever been provided with breast health education at the health facilities. Here, we found a significant negative correlation with BSE. $\left(\chi^{2}=45.59\right.$, $p=0.000)$. About $63.4 \%$ of the women who were not practicing BSE had not ever been given visual aids on breast cancer screening. However, advice on screening of breast cancer has 
a positive significant correlation on breast screening practices. However, $80 \%$ of respondents has not received advice for breast self-examination $\left(\chi^{2}=45.597, p=0.008\right)$. Regular breast health education should be provided at the maternity services and NCDs clinics to create awareness and improve breast screening practices. In this study, a majority of the respondents were not aware of breast health education, their role in prevention and control of breast cancer, and how to conduct BSE in their daily activities.

American Cancer Society reported that cultural and religious beliefs, challenging health needs, and deficiency in economic status are linked with ineffective breast cancer knowledge and deficiency health seeking behaviors (Cumber et al., 2017). The findings are in contrast to our results which revealed that majority of women (72\%) agreed that the major barrier was lack of clinics for breast screening. They also reported lack of knowledge on breast screening (49.2\%) and long distances to health facilities $(48.8 \%)$ were significant factors preventing them from adhering to breast screening. Shamseddine et al. (2010) reported that factors preventing women from performing breast cancer screening practices include the deficiency of breast cancer awareness due to poor health literacy and deficiency of education, deficiency of diagnostic programs, non-existence of governmental support, insufficient funds, and social obstacles to early detection due to low priority for female health-related issues. These

\section{REFERENCES}

Angahar, L. T. (2017). An overview of breast cancer epidemiology, risk factors, pathophysiology, and cancer risks reduction. MOJ Biology and Medicine, 1(4).

Bray, F., Ferlay, J., Soerjomataram, I., Siegel, R. L., Torre, L. A., \& Jemal, A. (2018). Global cancer statistics 2018: GLOBOCAN estimates of incidence and mortality worldwide for 36 cancers in 185 countries. CA: A Cancer Journal for Clinicians, 68(6), 394-424. https://doi.org/10.3322/caac.21492

Country-specific, I., Method, R., \& Total, M. (2020). 12952 209. 237, $1-2$.

Cumber, S. N., Nchanji, K. N., \& Tsoka-Gwegweni, J. M. (2017). Breast cancer among women in sub-Saharan Africa: Prevalence and a situational analysis. Southern African Journal of Gynaecological Oncology, 9(2), 35-37. https://doi.org/10.1080/20742835.2017.13914 67

Donkor, A., Wiafe, S., Yarney, J., Opoku, Y., Antwi, W., \& Kyei, K. A. (2015). iMedPub journals factors contributing to late presentation of breast cancer in Africa: A systematic literature review. Abstract. 1-10.

Feigin, V. L., Forouzanfar, M. H., Krishnamurthi, R., Mensah, G. A., Connor, M., Bennett, D. A., \& Murray, C. (2014). Global and regional burden of stroke during 1990-2010: Findings from the Global Burden of Disease Study 2010. The Lancet, 383(9913), 245-255.

Kohler, R. E., Gopal, S., Lee, C. N., Weiner, B. J., Reeve, B. B., \& Wheeler, S. B. (2017a). Breast cancer knowledge, behaviors, and preferences in Malawi: Implications for early detection interventions from a discrete choice experiment. Journal of Global Oncology, 3(5), 480-489. https://doi.org/10.1200/JGO.2016.005371

Kohler, R. E., Gopal, S., Lee, C. N., Weiner, B. J., Reeve, B. B., \& Wheeler, S. B. (2017b). Breast cancer knowledge, behaviors, and preferences in Malawi: Implications for early detection findings from low-income countries support our research findings which show many women are not aware of breast cancer screening practices and perhaps opportunities for breast screening are not available in their health centres.

\section{RECOMMENDATIONS}

There is a need to create and strengthen breast cancer awareness campaigns through all available media platforms like social media via short message services and breast camps for awareness in health facilities and schools. This will not only improve breast cancer screening practices, but also will improve breast cancer knowledge on its presentation, risk factors, and benefits of early detection and treatment for better health outcomes.

\section{CONCLUSION}

The study revealed deficiency in knowledge among respondents about breast cancer screening practice. Specially, the poor knowledge on breast cancer risk factors influenced breast cancer screening practices and screening testing. A large proportion of women have no source of information on breast cancer and its screening modalities. Among those who have information about breast cancer, radio was the main source used. Only a small proportion are occasionally practicing Breast Self-Examination.

interventions from a discrete choice experiment. Journal of Global Oncology, 3(5), 480-489. https://doi.org/10.1200/jgo.2016.005371

Majidi, A., Majidi, S., Salimzadeh, S., Pool, M. K.-, Sadjadi, A., Salimzadeh, H., \& Delavari, A. (2017). Cancer screening awareness and practice in a middle income country: A systematic review from Iran. Asian Pac J Cancer Prev, 18, 3187-3194. https://doi. org/10.22034/APJCP.2017.18.12.3187

Miesfeldt, S., Morse, E., Maegga, B., Joseph, , Gertrud, \& Miesfeldt, S. (2014). Breast cancer knowledge, beliefs, and screening practices among women seeking care at district hospitals in Dar es Salaam, Tanzania. Breast Cancer: Basic and Clinical Research, 73. https://doi. org/10.4137/BCBCR.S13745

Nambile, S., Nkeh, K., Tsoka-gwegweni, J. M., \& Africa, S. (2017). Breast cancer among women in sub-Saharan Africa: Prevalence and a situational analysis. Southern African Journal of Gynaecological Oncology, 9(2), 35-37. https://doi.org/10.1080/20742 835.2017.1391467

Pace, L. E., Dusengimana, J. M. V., Hategekimana, V., Habineza, H., Bigirimana, J. B., Tapela, N., ... \& Mpunga, T. (2016). Benign and malignant breast disease at Rwanda's first public cancer referral center. The oncologist, 21(5), 571.

Shamseddine, A., Tfayli, A., Temraz, S., \& Abou Mrad, R. (2010). Breast cancer in low- and middle-income countries: An emerging and challenging epidemic. Journal of Oncology, 2010. https://doi. org/10.1155/2010/490631

Tfayli, A., Temraz, S., Mrad, R. A., \& Shamseddine, A. (2010). Breast cancer in low- and middle-income countries: An emerging and challenging epidemic. Journal of Oncology, 2010. https://doi. org/10.1155/2010/490631

World Health Organization. (2020). WHO report on cancer: Setting priorities, investing wisely and providing care for all. 